International Journal of Pure and Applied Mathematics

Volume 103 No. $3 \quad 2015,567-586$

ISSN: 1311-8080 (printed version); ISSN: 1314-3395 (on-line version)

url: http://www.ijpam.eu

doi: http://dx.doi.org/10.12732/ijpam.v103i3.17

ijpam.eu

\title{
MULTIVARIATE HOMOGENEOUS MATRIX PADE-TYPE APPROXIMANTS AND PADE APPROXIMANTS
}

\author{
J. Abouir ${ }^{1 \S}$, B. Benouahmane ${ }^{2}$ \\ ${ }^{1,2}$ Laboratory LMANCS \\ Faculty of Science and Technology Mohammedia \\ University Hassan II - Casablanca \\ B.P. 146, Mohammedia, MOROCCO
}

\begin{abstract}
Matrix Padé-type approximants and Padé approximants has been studied by several authors in the scalar case. Some definitions in multivariate case have been introduced. In the current work, we define a multivariate homogeneous matrix-type approximants of which generating polynomials have shift. We consider higher-order approximants and introduce multivariate homogeneous matrix Padé approximants. We derive some recurrence relations for generating polynomials and associated polynomials. Finally, numerical example is given to illustrate our results.
\end{abstract}

AMS Subject Classification: 65D20, 41A20, 41A21, 33C45

Key Words: Homogeneous Padé approximants, matrix Padé approximants, matrix orthogonal polynomials, multivariate approximation

\section{Introduction}

The matrix Padé approximants has been studied for a long time by several authors $[8],[9],[13],[12],[14],[15]$ to cite juste a few. These approximants are used extensively in many fields : dynamic system control, multiport network synthesis, design of multi-input and multi-output digital filters, reduction of a high degree multivariable system.

Received: June 23, 2015

(C) 2015 Academic Publications, Ltd.

$\S_{\text {Correspondence author }}$ url: www.acadpubl.eu 
Bose and Basu [6] studied the existence, non uniqueness, and recursive computation of 2-D matrix Padé approximants with the inverse matrix. Gu [10] considered a bivariate matrix-value rational interpolant in Thiele-type continued fraction form with scalar denominator and matrix-value numerator.

Let $F$ be a rectangular matrix of size $r \times s$, defined by :

$$
F(x, y)=\left(\begin{array}{ccc}
f^{(1,1)}(x, y) & \ldots & f^{(1, s)}(x, y) \\
\vdots & & \vdots \\
f^{(r, 1)}(x, y) & \ldots & f^{(r, s)}(x, y)
\end{array}\right)
$$

It is assumed that the $f^{(k, l)}$ has a formal power series expansion. Therefore

$$
F(x, y)=\sum_{i, j=0}^{+\infty} C_{i, j} x^{i} y^{j}, \quad C_{i, j} \in \mathcal{M}_{r, s}
$$

where $\mathcal{M}_{r, s}$ is the space of rectangular matrices with real or complex coefficients of size $r \times s$.

C. D. Zheng et al. [14],[15] have defined the multivariate matrix Padé-type approximants and Padé approximants. They have generalized the concept of Brezinski in scalar cases [5]. Their definition is based on the bilinear functional $c$ acting on $\mathcal{P}$ ( the vector space of all bivariate polynomials whose coefficients are real or complex) defined from its moments by $c\left(u^{i} v^{j}\right)=C_{i, j}$. In the paper [1] we introduced a new family of multivariate matrix Padé approximants.

The paper is organized as follows. In Section 2, we recall the definition of multivariate homogeneous Padé approximants introduced by A. Cuyt in [7].

In Section 3, we give a definition of the multivariate homogeneous matrix Padé-type approximants. It generalizes directly the definition in [7].

In Section 4 we study the connexion between the homogeneous multivariate matrix Padé-type approximants and homogeneous multivariate matrix Padé approximants.

The recurrence relations are also established and proved for the generator polynomials and the associated polynomials in Section 5. In the last Section, we give a numerical example to illustrate our results.

We restrict our description to the bivariate case, only for notational convenience, although we may use the term multivariate in the text.

\section{Multivariate Homogeneous Padé Approximants}

The scalar homogeneous multivariate Padé-type approximants and Padé approximants are defined in $[2],[4],[7],[11]$. In this section, we recall the definition 
of approximants introduced by A. Cuyt in [7] of which the polynomials numerator and denominator have shift.

Let $f(x, y)$ be a formal power series:

$$
f(x, y)=\sum_{i, j=0}^{+\infty} d_{i j} x^{i} y^{j}
$$

For completeness we repeat that the multivariate homogeneous Padé approximant $[m-1 / m]_{f}$ is defined as an irreducible form $\frac{p}{q}$ with

$$
\begin{aligned}
p(x, y) & =\sum_{i+j=(m-1) m}^{(m-1) m+m-1} a_{i j} x^{i} y^{j} \\
q(x, y) & =\sum_{i+j=(m-1) m} b_{i j} x^{i} y^{j} \\
(f q-p)(x, y) & =\sum_{i+j=(m-1) m+2 m}^{\infty} e_{i j} x^{i} y^{j}
\end{aligned}
$$

One of the great advantage of this homogeneous definition is that it results in a unique irreducible form, whatever solution of (2.1), (2.2) and (2.3) is considered.

\section{Multivariate Homogeneous Matrix Padé-type Approximants}

Let $F$ be a rectangular matrix of size $r \times s$ with two variables, defined by:

$$
F(x, y)=\sum_{i, j=0}^{+\infty} C_{i, j} x^{i} y^{j}, \quad C_{i, j} \in \mathcal{M}_{r, s}
$$

This series can be written

$$
F(x, y)=\left(f^{(k, l)}(x, y)\right) \begin{aligned}
& 1 \leq k \leq r \\
& 1 \leq l \leq s
\end{aligned}
$$

It is assumed that the $f^{(k, l)}$ has a formal power series expansion.

$$
f^{(k, l)}(x, y)=\sum_{i, j=0}^{+\infty} c_{i, j}^{(k, l)} x^{i} y^{j}
$$


We introduce the notations bellow

$$
\begin{aligned}
& (x, y)=\left(\lambda_{1} z, \lambda_{2} z\right), \quad x, y, z \in \mathbb{C}, \quad \lambda=\left(\lambda_{1}, \lambda_{2}\right) \in \mathbb{C}^{2}, \quad\|\lambda\|=1 \\
& c_{i}^{(k, l)}(\lambda)=\sum_{j=0}^{i} c_{i-j, j}^{(k, l)} \lambda_{1}^{i-j} \lambda_{2}^{j} \\
& C_{i}(\lambda)=\left(c_{i}^{(k, l)}(\lambda)\right) \quad 1 \leq k \leq r \\
& 1 \leq l \leq s
\end{aligned}
$$

Now the series can also be formally viewed as

$$
F(x, y)=F_{\lambda}(z)=\sum_{i=0}^{+\infty} C_{i}(\lambda) z^{i}=\left(\sum_{i=0}^{+\infty} c_{i}^{(k, l)}(\lambda) z^{i}\right) \begin{aligned}
& 1 \leq k \leq r \\
& 1 \leq l \leq s
\end{aligned}
$$

For $n, m \in \mathbb{N}$, we consider an arbitrary function $V_{n, m}(x, y)$ of the form

$$
\begin{aligned}
V_{n, m}(x, y)=\mathcal{V}_{n, m}(z) & =\sum_{i=0}^{m} b_{n m+m-i}(\lambda) z^{i} \\
& =\sum_{i=0}^{m}\left(\sum_{j=0}^{n m+m-i} b_{n m+m-i-j, j} \lambda_{1}^{n m+m-i-j} \lambda_{2}^{j}\right)^{z^{i}}
\end{aligned}
$$

$\mathcal{V}_{n, m}(z)$ is a polynomial of degree $m$ in $z$ and its coefficients are homogeneous polynomials. Let $W_{n, m}$ be defined by

$$
W_{n, m}(x, y)=\mathcal{W}_{n, m}(z)=\Gamma_{n, m}\left(\frac{\mathcal{V}_{n, m}(u)-\mathcal{V}_{n, m}(z)}{u-z}\right)
$$

where the linear functional $\Gamma_{n, m}$ acting on the space of bivariate polynomials in variable $u$, as

$$
\Gamma_{n, m}\left(u^{i}\right)=\left(\Gamma_{n, m}^{(k, l)}\left(u^{i}\right)\right) \begin{aligned}
& 1 \leq k \leq r \\
& 1 \leq l \leq s
\end{aligned}=\left(c_{n-m+1+i}^{(k, l)}(\lambda)\right) \begin{aligned}
& 1 \leq k \leq r \\
& 1 \leq l \leq s
\end{aligned}
$$

with the convention that $c_{i}^{(k, l)}(\lambda)=0$ if $i<0$.

Proposition. The polynomial $\mathcal{W}_{n, m}$ is of the form

$$
\mathcal{W}_{n, m}(z)=\sum_{i=0}^{m-1} A_{n m+n-i}(\lambda) z^{i}=\left(\sum_{i=0}^{m-1} a_{n m+n-i}^{(k, l)}(\lambda) z^{i}\right) \begin{gathered}
1 \leq k \leq r \\
1 \leq l \leq s
\end{gathered}
$$


Proof. $\mathcal{W}_{n, m}$ is the matrix polynomial of degree $m-1$ in $z$.

$$
\begin{aligned}
\frac{\mathcal{V}_{n, m}(u)-\mathcal{V}_{n, m}(z)}{u-z}= & \sum_{i=1}^{m} b_{n m+m-i}(\lambda) \frac{u^{i}-z^{i}}{u-z} \\
& =\sum_{i=1}^{m} b_{n m+m-i}(\lambda) \sum_{\mu=0}^{i-1} u^{i-1-\mu} z^{\mu} \\
\mathcal{W}_{n, m}(z)= & \Gamma_{n, m}\left(\frac{\mathcal{V}_{n, m}(u)-\mathcal{V}_{n, m}(z)}{u-z}\right) \\
= & \sum_{i=1}^{m} b_{n m+m-i}(\lambda) \sum_{\mu=0}^{i-1} \Gamma_{n, m}\left(u^{i-1-\mu}\right) z^{\mu} \\
= & \sum_{i=1}^{m} b_{n m+m-i}(\lambda) \sum_{\mu=0}^{i-1} C_{n-m+i-\mu}(\lambda) z^{\mu}
\end{aligned}
$$

If these sums were developed, is obtained by identifying the coefficients matrix $A_{n m+n-i}, \quad 0 \leq i \leq m-1$, of the polynomial $\mathcal{W}_{n, m}(z)$.

More precisely

$$
A_{n m+n-i}^{(k, l)}=\sum_{j=0}^{m-1-i} b_{n m+m-1-i-j}(\lambda) C_{n-m+1+j}(\lambda) .
$$

Let

$$
\begin{gathered}
\widetilde{V}_{n, m}(x, y)=\widetilde{\mathcal{V}}_{n, m}(z)=z^{n m+m} \mathcal{V}_{n, m}\left(z^{-1}\right)=\sum_{i=0}^{m} b_{n m+i}(\lambda) z^{n m+i} \\
\widetilde{W}_{n, m}(x, y)=\widetilde{\mathcal{W}}_{n, m}(z)=z^{n m+m-1} \mathcal{W}_{n, m}\left(z^{-1}\right)=\sum_{i=0}^{m-1} A_{n m+n-i}(\lambda) z^{n m+m-1-i}
\end{gathered}
$$
by:

Define the multivariate homogeneous matrix Padé-type approximant $(n / m)_{F}^{H}$

$$
(n / m)_{F}^{H}(x, y)=\sum_{i=0}^{n-m} C_{i}(\lambda) z^{i}+z^{n-m+1} \frac{\widetilde{\mathcal{W}}_{n, m}(z)}{\widetilde{\mathcal{V}}_{n, m}(z)}
$$


The numerator and denominator of this fraction are polynomials in $x$ and $y$ containing only terms of total degree respectively between $n m$ and $n m+n$, and between $n m$ and $n m+m$.

It is assumed that the sum is null if $n<m$.

Let $\mathcal{V}_{m}(z)=\sum_{i=0}^{m} b_{m^{2}-i}^{(m)}(\lambda) z^{i}$ be the generator polynomial of the $(m-1 / m)_{F}^{H}$. We have:

Theorem 1.

$$
\begin{aligned}
F(x, y)-(m-1 / m)_{F}^{H}(x, y) & =\frac{z^{m^{2}}}{\widetilde{\mathcal{V}}_{m}(z)} \Gamma\left(\frac{\mathcal{V}_{m}(u)}{1-u z}\right) \\
& =\left(\frac{z^{m^{2}}}{\widetilde{\mathcal{V}}_{m}(z)} \Gamma^{(k, l)}\left(\frac{\mathcal{V}_{m}(u)}{1-u z}\right)\right) \begin{array}{l}
1 \leq k \leq r \\
1 \leq l \leq s
\end{array}
\end{aligned}
$$

Proof. We note $\Gamma_{m-1, m}=\Gamma$.

Firstly

$$
F(x, y)=\sum_{i=0}^{+\infty} C_{i}(\lambda) z^{i}=\Gamma\left(\frac{1}{1-u z}\right)=\frac{z^{m^{2}}}{\widetilde{\mathcal{V}}_{m}(z)} \Gamma\left(\frac{\mathcal{V}_{m}\left(z^{-1}\right)}{1-u z}\right)
$$

Secondly

$$
\begin{aligned}
(m-1 / m)_{F}^{H}(x, y) & =\frac{\widetilde{\mathcal{W}}_{m-1}(z)}{\widetilde{\mathcal{V}}_{m}(z)} \\
& =\frac{z^{m^{2}-1}}{\widetilde{\mathcal{V}}_{m}(z)} \Gamma\left(\frac{\mathcal{V}_{m}(u)-\mathcal{V}_{m}\left(z^{-1}\right)}{u-z^{-1}}\right) \\
& =\frac{z^{m^{2}}}{\widetilde{\mathcal{V}}_{m}(z)} \Gamma\left(\frac{\mathcal{V}_{m}(u)-\mathcal{V}_{m}\left(z^{-1}\right)}{u z-1}\right)
\end{aligned}
$$

Then

$$
\begin{aligned}
F(x, y)-(m-1 / m)_{F}^{H}(x, y) & =\frac{z^{m^{2}}}{\widetilde{\mathcal{V}}_{m}(z)} \Gamma\left(\frac{\mathcal{V}_{m}(u)}{1-u z}\right) \\
& =\left(\frac{z^{m^{2}}}{\widetilde{\mathcal{V}}_{m}(z)} \Gamma^{(k, l)}\left(\frac{\mathcal{V}_{m}(u)}{1-u z}\right)\right) \begin{array}{l}
\begin{array}{l}
1 \leq k \leq r \\
1 \leq l \leq s
\end{array} \\
1 \leq l \leq s
\end{array}
\end{aligned}
$$




\section{Multivariate Homogeneous Matrix Padé Approximants}

In this part, we will assume that orthogonality conditions are met, in order to reach high order estimates. These conditions will be used to calculate the denominator $\tilde{\mathcal{V}}_{m}(z)$ of the matrix Padé approximant $[m-1 / m]_{F}^{H}$.

The level of the approximation is determined by the theorem bellow:

Theorem 2. Let $F_{\lambda}(z)=\sum_{i=0}^{+\infty} C_{i}(\lambda) z^{i}$ given in (3.3), we have

$$
\tilde{\mathcal{V}}_{m}(z) F_{\lambda}(z)-\tilde{\mathcal{W}}_{m-1}(z)=O\left(z^{m^{2}}\right)
$$

Proof. By using the relation (3.6), we have

$$
\begin{aligned}
\tilde{\mathcal{V}}_{m}(z) F_{\lambda}(z)-\tilde{\mathcal{W}}_{m-1}(z) & =z^{m^{2}} \Gamma\left(\frac{\mathcal{V}_{m}(u)}{1-u z}\right) \\
& =z^{m^{2}} \Gamma\left(\mathcal{V}_{m}(u) \sum_{i=0}^{+\infty} u^{i} z^{i}\right) \\
& =z^{m^{2}} \Gamma\left(\left(\sum_{j=0}^{m} b_{m^{2}-j}^{(m)}(\lambda) u^{j}\right) \sum_{i=0}^{+\infty} u^{i} z^{i}\right) \\
& =z^{m^{2}} \sum_{i=0}^{+\infty} \Gamma\left(\sum_{j=0}^{m} b_{m^{2}-j}^{(m)}(\lambda) u^{i+j}\right)^{z^{i}} \\
& =z^{m^{2}} \sum_{i=0}^{+\infty}\left(\sum_{j=0}^{m} b_{m^{2}-j}^{(m)}(\lambda) C_{i+j}(\lambda) z^{z^{i}}\right. \\
& =O\left(z^{m^{2}}\right)
\end{aligned}
$$

So, we obtain:

$$
\begin{gathered}
F(x, y)-(m-1 / m)_{F}^{H}(x, y)=\frac{z^{m^{2}}}{\widetilde{\mathcal{V}}_{m}(z)} \Gamma\left(\frac{\mathcal{V}_{m}(u)}{1-u z}\right) \\
=\frac{z^{m^{2}}}{\widetilde{\mathcal{V}}_{m}(z)}\left(\Gamma^{(k, l)}\left(\frac{\mathcal{V}_{m}(u)}{1-u z}\right)\right) \begin{array}{l}
1 \leq k \leq r \\
1 \leq l \leq s
\end{array}
\end{gathered}
$$




$$
\begin{aligned}
= & \frac{z^{m^{2}}}{\widetilde{\mathcal{V}}_{m}(z)}\left(\Gamma^{(k, l)}\left(\mathcal{V}_{m}(u)\right)+\Gamma^{(k, l)}\left(u \mathcal{V}_{m}(u)\right) z+\cdots\right. \\
& \left.+\Gamma^{(k, l)}\left(u^{m-1} \mathcal{V}_{m}(u)\right) z^{m-1}+z^{m} \Gamma^{(k, l)}\left(\frac{u^{m} \mathcal{V}_{m}(u)}{1-u z}\right)\right) \begin{array}{l}
1 \leq k \leq r \\
1 \leq l \leq s
\end{array}
\end{aligned}
$$

We write

$$
\left\{\begin{array}{l}
m=p_{m} \times r s+n_{m} \\
n_{m}=q_{m} \times s+t_{m}
\end{array}\right.
$$

where $p_{m}$ and $n_{m}$ are respectively the quotient and rest of the Euclidean division of $m$ by $r s$. Also $q_{m}$ and $t_{m}$ are respectively the quotient and rest of the Euclidean division of $n_{m}$ by $s$.

Let $\alpha_{m}$ be the integer:

$$
\alpha_{m}=\frac{m(m-1)+\left(p_{m}+1\right)\left(n_{m}+m\right)}{2},
$$

and $\mathcal{V}_{m}(z)=\sum_{i=0}^{m} b_{\alpha_{m}-i}^{(m)}(\lambda) z^{i}$ be the polynomial generator of $(m-1 / m)_{F}^{H}$ where $b_{\alpha_{m}-i}^{(m)}(\lambda)=\sum_{j=0}^{\alpha_{m}-i} b_{\alpha_{m}-i-j, j}^{(m)} \lambda_{1}^{\alpha_{m}-i-j} \lambda_{2}^{j}$.

The approximation error is given as follows:

$$
\begin{aligned}
& F(x, y)-(m-1 / m)_{F}^{H}(x, y)=\frac{z^{\alpha_{m}}}{\widetilde{\mathcal{V}}_{m}(z)} \Gamma\left(\frac{\mathcal{V}_{m}(u)}{1-u z}\right) \\
= & \frac{z^{\alpha_{m}}}{\widetilde{\mathcal{V}}_{m}(z)}\left(\Gamma^{(k, l)}\left(\frac{\mathcal{V}_{m}(u)}{1-u z}\right)\right) \begin{array}{l}
1 \leq k \leq r \\
1 \leq l \leq s
\end{array} \\
= & \frac{z^{\alpha_{m}}}{\widetilde{\mathcal{V}}_{m}(z)}\left\{\Gamma^{(k, l)}\left(\mathcal{V}_{m}(u)\right)+\Gamma^{(k, l)}\left(u \mathcal{V}_{m}(u)\right) z+\cdots+\Gamma^{(k, l)}\left(u^{p_{m}-1} \mathcal{V}_{m}(u)\right) z^{p_{m}-1}\right. \\
+ & \left.\Gamma^{(k, l)}\left(u^{p_{m}} \mathcal{V}_{m}(u)\right) z^{p_{m}}+\Gamma^{(k, l)}\left(\frac{u^{p_{m}+1} \mathcal{V}_{m}(u)}{1-u z}\right) z^{p_{m}+1}\right\} \begin{array}{l}
\substack{1 \leq k \leq r \\
1 \leq l \leq s}
\end{array}
\end{aligned}
$$

If we choose the polynomial $\mathcal{V}_{m}$ in the way that $\left(\Gamma^{(k, l)}\left(u^{i} \mathcal{V}_{m}(u)\right)\right) \quad 1 \leq k \leq r=$ $1 \leq l \leq s$

$0, i=0, \ldots, j-1 \leq p_{m}-1$, we will obtain an approximation which is a $O\left(z^{\alpha_{m}+j}\right)$ 
as the $j$ first terms of the previous expression will vanish.

The $\mathcal{V}_{m}$ polynomial has $(m+1) \alpha_{m}-\frac{m(m-1)}{2}+1$ arbitrary coefficients. It is almost entirely determined by the conditions:

$$
\begin{aligned}
& \Gamma^{(k, l)}\left(u^{j} \mathcal{V}_{m}(u)\right)=0,1 \leq k \leq r, 1 \leq l \leq s, 0 \leq j \leq p_{m}-1 \\
& \Gamma^{(k, l)}\left(u^{p_{m}} \mathcal{V}_{m}(u)\right)=0,1 \leq k \leq q_{m}, 1 \leq l \leq s \\
& \Gamma^{\left(q_{m}+1, l\right)}\left(u^{p_{m}} \mathcal{V}_{m}(u)\right)=0,1 \leq l \leq t_{m}
\end{aligned}
$$

Then, we will have:

$$
\begin{gathered}
F(x, y)-(m-1 / m)_{F}^{H}(x, y)=\frac{z^{\alpha_{m}+p_{m}}}{\widetilde{\mathcal{V}}_{m}(z)}\left(\Gamma^{(k, l)}\left(u^{p_{m}} \mathcal{V}_{m}(u)\right) \begin{array}{l}
1 \leq k \leq r \\
1 \leq l \leq s
\end{array}\right. \\
+\frac{z^{\alpha_{m}+p_{m}+1}}{\widetilde{\mathcal{V}}_{m}(z)}\left(\Gamma^{(k, l)}\left(\frac{u^{p_{m}+1} \mathcal{V}_{m}(u)}{1-u z}\right)\right) \begin{array}{l}
\begin{array}{l}
1 \leq k \leq r \\
1 \leq l \leq s
\end{array}
\end{array}
\end{gathered}
$$

where the $q_{m}$ first lines and the $t_{m}$ first components of the line $q_{m}+1$ of the matrix $\left(\Gamma^{(k, l)}\left(u^{p_{m}} \mathcal{V}_{m}(u)\right)\right) \quad 1 \leq k \leq r$ are null.

$$
1 \leq l \leq s
$$

The conditions (4.2), (4.3) and (4.4) are represented in the systems follows:

$$
\begin{aligned}
& \sum_{i=0}^{m} b_{\alpha_{m}-i}^{(m)}(\lambda) c_{i+j}^{(k, l)}(\lambda)=0,1 \leq k \leq r, 1 \leq l \leq s, 0 \leq j \leq p_{m}-1 \\
& \sum_{i=0}^{m} b_{\alpha_{m}-i}^{(m)}(\lambda) c_{i+p_{m}}^{(k, l)}(\lambda)=0,1 \leq k \leq q_{m}, 1 \leq l \leq s \\
& \sum_{i=0}^{m} b_{\alpha_{m}-i}^{(m)}(\lambda) c_{i+p_{m}}^{\left(q_{m}+1, l\right)}(\lambda)=0,1 \leq l \leq t_{m}
\end{aligned}
$$

We have a total $(m+1) \alpha_{m}-\frac{(m-1) m}{2}$ equations and $(m+1) \alpha_{m}-$ $\frac{(m-1) m}{2}+1$ unknown variables.

With the $C_{i}(\lambda)$, we can define the polynomial Hankel determinants generalizing the determinants as in [1],

$$
H_{0}^{(j)}(\lambda)=1
$$


and for $m \geq 1$

$H_{m}^{(j)}(\lambda)=$

$\begin{array}{cccc}c_{p_{j}}^{\left(q_{j}+1, t_{j}+1\right)}(\lambda) & c_{p_{j}+1}^{\left(q_{j}+1, t_{j}+1\right)}(\lambda) & \ldots & c_{p_{j}+m-1}^{\left(q_{j}+1, t_{j}+1\right)}(\lambda) \\ c_{p_{j+1}}^{\left(q_{j+1}+1, t_{j+1}+1\right)}(\lambda) & c_{p_{j+1}+1}^{\left(q_{j+1}+1, t_{j+1}+1\right)}(\lambda) & \ldots & c_{p_{j+1}+m-1}^{\left(q_{j+1}+1, t_{j+1}+1\right)}(\lambda) \\ \vdots & \vdots & \ldots & \vdots \\ c_{p_{j+m-1}}^{\left(q_{j+m-1}+1, t_{j+m-1}+1\right)}(\lambda) & c_{p_{j+m-1}+1}^{\left(q_{j+m-1}+1, t_{j+m-1}+1\right)}(\lambda) & \ldots & c_{p_{j+m-1}+m-1}^{\left(q_{j+m-1}+1, t_{j+m-1}+1\right)}(\lambda)\end{array}$

If for every $m \geq 0, H_{m}^{(j)}(\lambda) \not \equiv 0$, we say that the linear functional $\Gamma=$ $\left(\Gamma^{(k, l)}\right) \quad 1 \leq k \leq r$ is well defined.

$1 \leq l \leq s$

Remark. If we suppose that the linear functional $\Gamma$ is well defined, the linear systems (4.6), (4.7) and (4.8) that gives $b_{\alpha_{m}-i}^{(m)}(\lambda)$ coefficients has a solution giving by:

$$
\begin{aligned}
& \mathcal{V}_{0}(z)=1
\end{aligned}
$$

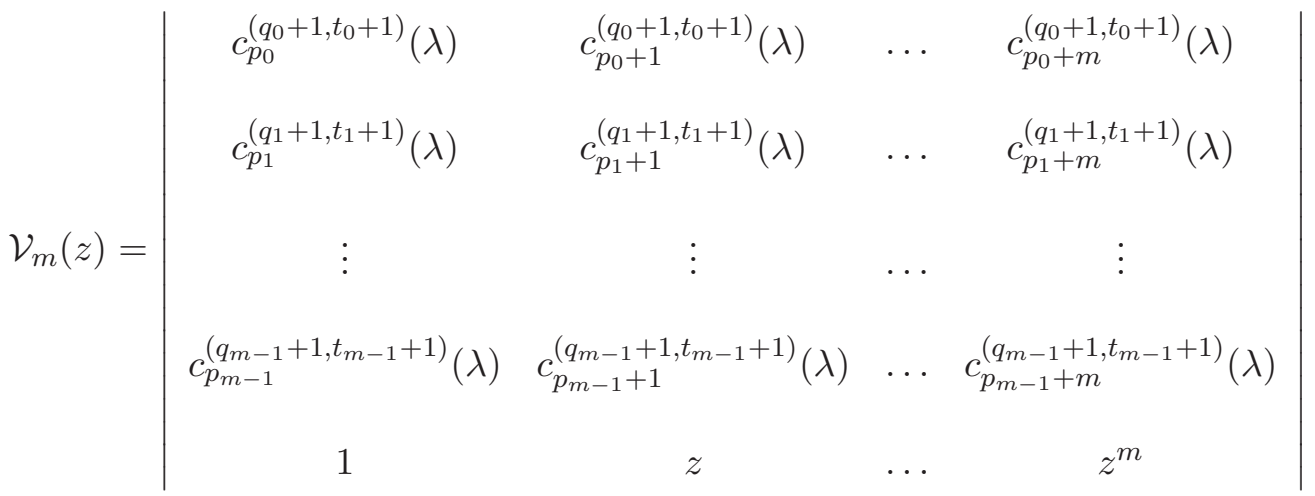

So we have

$$
\mathcal{W}_{m-1}(z)=\Gamma\left(\frac{\mathcal{V}_{m}(u)-\mathcal{V}_{m}(z)}{u-z}\right)
$$




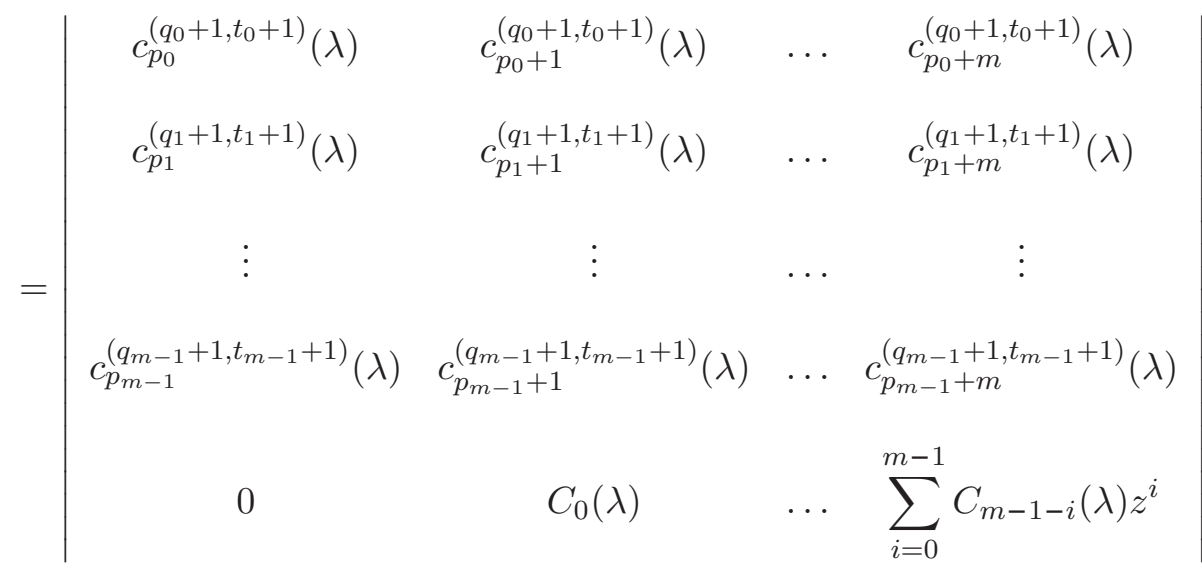

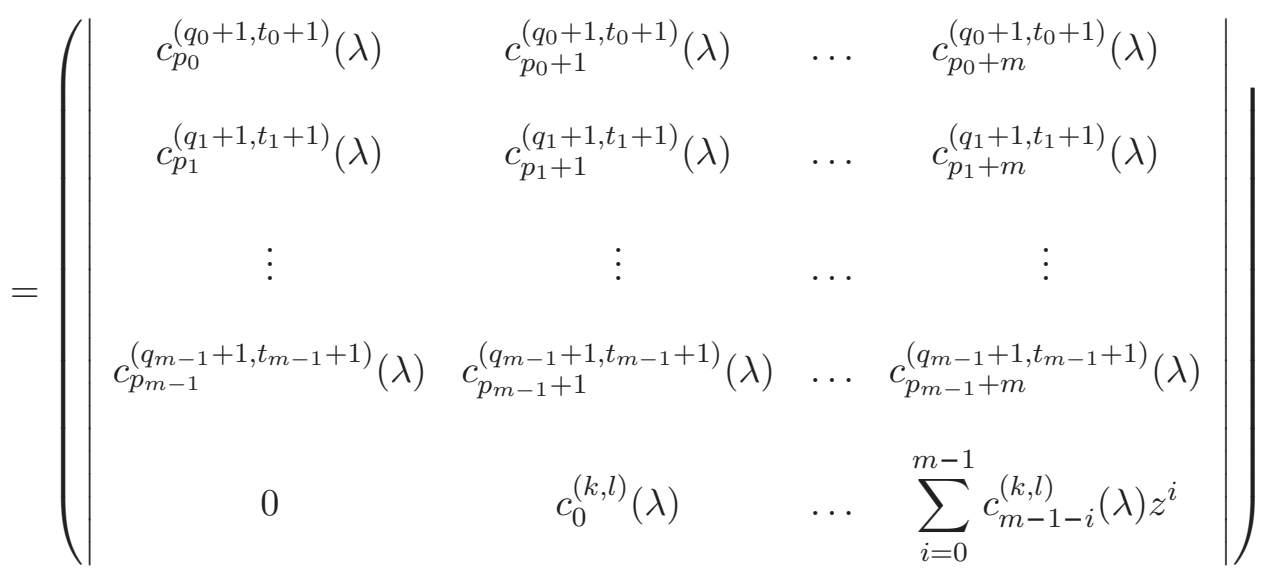

$1 \leq k \leq r$ $1 \leq l \leq s$

$$
\begin{aligned}
& \tilde{\mathcal{V}}_{m}(z)=z^{m} \mathcal{V}_{m}\left(z^{-1}\right) \\
& =\left|\begin{array}{cccc}
c_{p_{0}}^{\left(q_{0}+1, t_{0}+1\right)}(\lambda) & c_{p_{0}+1}^{\left(q_{0}+1, t_{0}+1\right)}(\lambda) & \ldots & c_{p_{0}+m}^{\left(q_{0}+1, t_{0}+1\right)}(\lambda) \\
c_{p_{1}}^{\left(q_{1}+1, t_{1}+1\right)}(\lambda) & c_{p_{1}+1}^{\left(q_{1}+1, t_{1}+1\right)}(\lambda) & \ldots & c_{p_{1}+m}^{\left(q_{1}+1, t_{1}+1\right)}(\lambda) \\
\vdots & \vdots & \ldots & \vdots \\
c_{p_{m-1}}^{\left(q_{m-1}+1, t_{m-1}+1\right)}(\lambda) & c_{p_{m-1}+1}^{\left(q_{m-1}+1, t_{m-1}+1\right)}(\lambda) & \ldots & c_{p_{m-1}+m}^{\left(q_{m-1}+1, t_{m-1}+1\right)}(\lambda) \\
z^{m} & z^{m-1} & \ldots & 1
\end{array}\right|
\end{aligned}
$$


Likewise

$$
\tilde{\mathcal{W}}_{m-1}(z)=z^{m-1} \mathcal{W}_{m-1}\left(z^{-1}\right)
$$

$$
=\left|\begin{array}{cccc}
c_{p_{0}}^{\left(q_{0}+1, t_{0}+1\right)}(\lambda) & c_{p_{0}+1}^{\left(q_{0}+1, t_{0}+1\right)}(\lambda) & \ldots & c_{p_{0}+m}^{\left(q_{0}+1, t_{0}+1\right)}(\lambda) \\
c_{p_{1}}^{\left(q_{1}+1, t_{1}+1\right)}(\lambda) & c_{p_{1}+1}^{\left(q_{1}+1, t_{1}+1\right)}(\lambda) & \ldots & c_{p_{1}+m}^{\left(q_{1}+1, t_{1}+1\right)}(\lambda) \\
\vdots & \vdots & \ldots & \vdots \\
c_{p_{m-1}}^{\left(q_{m-1}+1, t_{m-1}+1\right)}(\lambda) & c_{p_{m-1}+1}^{\left(q_{m-1}+1, t_{m-1}+1\right)}(\lambda) & \ldots & c_{p_{m-1}+m}^{\left(q_{m-1}+1, t_{m-1}+1\right)}(\lambda) \\
z^{m} N_{-1}(z) & z^{m-1} N_{0}(z) & \ldots & N_{m-1}(z)
\end{array}\right|,
$$

where $N_{i}(z)=\sum_{k=0}^{i} C_{k}(\lambda) z^{k}$ and $N_{-1}(z) \equiv 0$.

We notice that $\tilde{\mathcal{W}}_{m-1}(z)$ is a polynomial in $z$ of degree $m-1$. We divide the $j$ th column $(j=1, \ldots, m+1)$ of the two previous determinants by $z^{m-j+1}$ and we multiply the $i=1, \ldots, m$ line by $z^{m}$.

The quotient of the two new determinants is the homogeneous matrix Padé approximant $[m-1 / m]_{F}^{H}$

$$
[m-1 / m]_{F}^{H}\left(\lambda_{1} z, \lambda_{2} z\right)=\frac{1}{\tilde{\mathcal{V}}_{m}(z)} \cdot \tilde{\mathcal{W}}_{m-1}(z)
$$




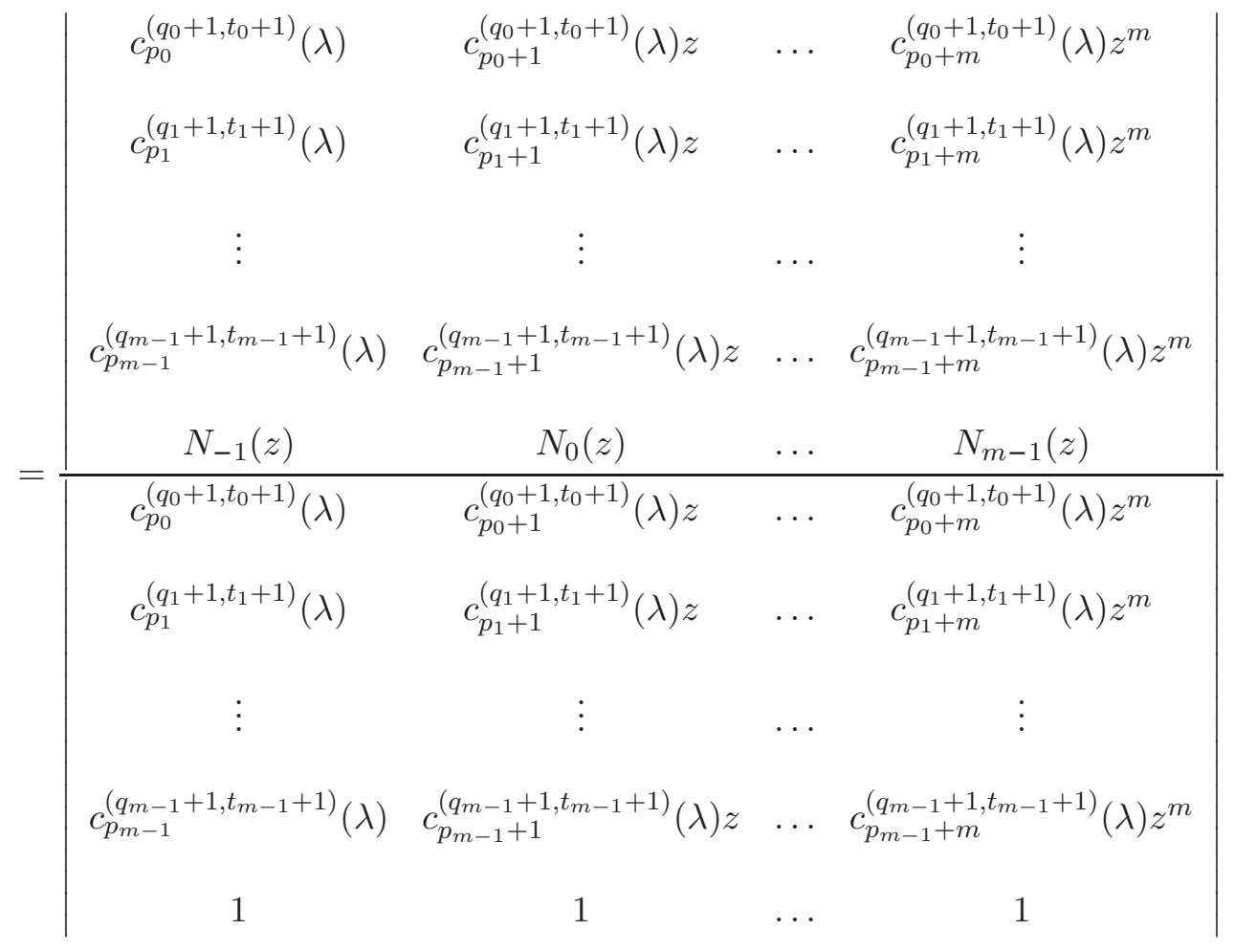

\section{Recurrence Relations}

In the sequel, we shall assume that the linear functional $\Gamma$ is well defined.

Lemma. The polynomials $\left\{\mathcal{V}_{m}\right\}$ are linearly independent in $\mathbb{C}(\lambda)[z]$, the space of rational functions in the variable $z$ with coefficients being complexvalued bivariate polynomials in $\lambda$.

Proof. Let's suppose the existence of a combinaison of factors $\eta_{0}(\lambda), \eta_{1}(\lambda), \ldots \in$ $\mathbb{C}(\lambda)$ as

$$
\sum_{i=0}^{+\infty} \eta_{i}(\lambda) \mathcal{V}_{i}(z)=0 \quad \forall z \in \mathbb{C}
$$

Also for $j \geq 0$, we obtain

$$
\sum_{i=0}^{+\infty} \eta_{i}(\lambda) \Gamma^{\left(q_{j}+1, t_{j}+1\right)}\left(z^{p_{j}} \mathcal{V}_{i}(z)\right) \equiv 0
$$


Taking (4.2) into account, we obtain for $j \geq 0$

$$
\sum_{i=0}^{j} \eta_{i}(\lambda) \Gamma^{\left(q_{j}+1, t_{j}+1\right)}\left(z^{p_{j}} \mathcal{V}_{i}(z)\right) \equiv 0
$$

For $j=0$, the combinaison is reduced to

$$
\eta_{0}(\lambda) \Gamma^{(1,1)}\left(\mathcal{V}_{0}(z)\right) \equiv 0
$$

This result is that $\eta_{0}(\lambda)=0$ because $\Gamma^{(1,1)}\left(\mathcal{V}_{0}(z)\right)=H_{1}^{(0)}(\lambda) \not \equiv 0$.

For $j \geq 1$ the proof that $\eta_{j}(\lambda)=0$ is by induction.

Theorem 3. The polynomials $\mathcal{V}_{m}(z)$ and $\mathcal{W}_{m}(z)$ satisfy the recurrence relations:

$$
\begin{gathered}
z \mathcal{V}_{m}(z)=\sum_{i=\max (0, m-r s)}^{m+1} \varphi_{i}^{(m+1)}(\lambda) \mathcal{V}_{i}(z), \quad \mathcal{V}_{0}(z)=1 \quad \mathcal{W}_{-1}(z)=0 \\
z \mathcal{W}_{m-1}(z)+\Gamma\left(\mathcal{V}_{m}(z)\right)=\sum_{i=\max (0, m-r s)}^{m+1} \varphi_{i}^{(m+1)}(\lambda) \mathcal{W}_{i-1}(z), \quad
\end{gathered}
$$

The $\varphi_{i}^{(m+1)}(\lambda), 0 \leq i \leq m$, are computed using the triangular system:

$$
\Gamma^{\left(q_{j}+1, t_{j}+1\right)}\left(z^{p_{j}+1} \mathcal{V}_{m}(z)\right)=\sum_{i=0}^{j} \varphi_{i}^{(m+1)}(\lambda) \Gamma^{\left(q_{j}+1, t_{j}+1\right)}\left(z^{p_{j}} \mathcal{V}_{i}(z)\right), \quad 0 \leq j \leq m
$$

Proof. The polynomials $z \mathcal{V}_{m}(z)$ can be written as linear combinaison:

$$
z \mathcal{V}_{m}(z)=\sum_{i=0}^{m+1} \varphi_{i}^{(m+1)}(\lambda) \mathcal{V}_{i}(z)
$$

where $\varphi_{i}^{(m+1)}(\lambda)$ are the rational functions of the variable $\lambda$.

We multiply left and right hand sides with $z^{p_{j}}$ and apply the linear functional $\Gamma$ to obtain:

$$
\begin{gathered}
\varphi_{i}^{(m+1)}(\lambda)=0, i=0, \ldots, m-r s-1(\text { if } m \geq r s+1) \\
\Gamma^{\left(q_{j}+1, t_{j}+1\right)}\left(z^{p_{j}+1} \mathcal{V}_{m}(z)\right)=\sum_{i=0}^{j} \varphi_{i}^{(m+1)}(\lambda) \Gamma^{\left(q_{j}+1, t_{j}+1\right)}\left(z^{p_{j}} \mathcal{V}_{i}(z)\right), \quad 0 \leq j \leq m
\end{gathered}
$$


On the other hand, we have

$$
\mathcal{V}_{m}(z)=H_{m}^{(0)}(\lambda) z^{m}+\ldots,
$$

So that consequently

$$
H_{m}^{(0)}(\lambda)=\varphi_{m+1}^{(m+1)}(\lambda) H_{m+1}^{(0)}(\lambda)
$$

For the associated polynomials $\left\{\mathcal{W}_{m}(z)\right\}$ we have, using

$$
\begin{aligned}
& \frac{u \mathcal{V}_{m}(u)-z \mathcal{V}_{m}(z)}{u-z}=z \frac{\mathcal{V}_{m}(u)-\mathcal{V}_{m}(z)}{u-z}+\mathcal{V}_{m}(u), \\
& \Gamma\left(z \frac{\mathcal{V}_{m}(u)-\mathcal{V}_{m}(z)}{u-z}+\mathcal{V}_{m}(u)\right)=\sum_{i=\max (0, m-r s)}^{m+1} \varphi_{i}^{(m+1)}(\lambda) \Gamma\left(\frac{\mathcal{V}_{i}(u)-\mathcal{V}_{i}(z)}{u-z}\right)
\end{aligned}
$$

which gives the expected results.

To compute the polynomial Hankel determinants, we need the following theorem proved in [3].

Theorem 4. Let $A$ be a matrix, and let $A_{\mu k}$ denote the matrix with row $\mu$ and column $k$ deleted. Also let $A_{\mu \nu, k l}$ denote the matrix $A$ with rows $\mu$ and $\nu$ and columns $k$ and $l$ deleted. Provided $\mu<\nu$ and $k<l$,

$$
\operatorname{det} A \operatorname{det} A_{\mu \nu, k l}=\operatorname{det} A_{\mu k} \operatorname{det} A_{\nu l}-\operatorname{det} A_{\mu l} \operatorname{det} A_{\nu k} \text {. }
$$

Theorem 5. The polynomial Hankel determinants $\left\{H_{m}^{(j)}\right\}$ satisfies the recurrence relation

$$
H_{m+1}^{(j)}(\lambda) H_{m-1}^{(r s+j+1)}(\lambda)=H_{m}^{(j)}(\lambda) H_{m}^{(r s+j+1)}(\lambda)-H_{m}^{(j+1)}(\lambda) H_{m}^{(r s+j)}(\lambda)
$$

for $m, j=1,2, \ldots$

$$
H_{-1}^{(j)}(\lambda)=0, \quad H_{0}^{(j)}(\lambda)=1 .
$$

Proof. Let $A$ denote the matrix

$$
A=\left(\begin{array}{cccc}
c_{p_{j}}^{\left(q_{j}+1, t_{j}+1\right)}(\lambda) & c_{p_{j}+1}^{\left(q_{j}+1, t_{j}+1\right)}(\lambda) & \ldots & c_{p_{j}+m}^{\left(q_{j}+1, t_{j}+1\right)}(\lambda) \\
c_{p_{j+1}}^{\left(q_{j+1}+1, t_{j+1}+1\right)}(\lambda) & c_{p_{j+1}+1}^{\left(q_{j+1}+1, t_{j+1}+1\right)}(\lambda) & \ldots & c_{p_{j+1}+m}^{\left(q_{j+1}+1, t_{j+1}+1\right)}(\lambda) \\
\vdots & \vdots & \ldots & \vdots \\
c_{p_{j+m}}^{\left(q_{j+m}+1, t_{j+m}+1\right)}(\lambda) & c_{p_{j+m}+1}^{\left(q_{j+m}+1, t_{j+m}+1\right)}(\lambda) & \ldots & c_{p_{j+m}+m}^{\left(q_{j+m}+1, t_{j+m}+1\right)}(\lambda)
\end{array}\right)
$$


We take $\mu=1, \nu=m+1, k=1$ and $l=m+1$. The theorem is a consequence of the previous theorem of fact that

$\operatorname{det} A=H_{m+1}^{(j)}(\lambda), \quad \operatorname{det} A_{\mu \nu, k l}=H_{m-1}^{(r s+j+1)}(\lambda), \quad \operatorname{det} A_{\mu k}=H_{m}^{(r s+j+1)}(\lambda)$,

$$
\operatorname{det} A_{\nu l}=H_{m}^{(j)}(\lambda), \quad \operatorname{det} A_{\mu l}=H_{m}^{(j+1)}(\lambda), \quad \operatorname{det} A_{\nu k}=H_{m}^{(r s+j)}(\lambda)
$$

and the follows relations satisfies by $p_{j}, q_{j}, n_{j}$ and $t_{j}$ introduced in (4.1),

$$
\begin{gathered}
p_{j+k . r s}=p_{j}+k, \quad n_{j+k . r s}=n_{j} \\
q_{j+k . r s}=q_{j}+k, \quad t_{j+k . r s}=t_{j}
\end{gathered}
$$

By the previous theorem, we deduce the recursive relation:

$$
H_{m+1}^{(j)}(\lambda) H_{m-1}^{(r s+j+1)}(\lambda)=H_{m}^{(j)}(\lambda) H_{m}^{(r s+j+1)}(\lambda)-H_{m}^{(j+1)}(\lambda) H_{m}^{(r s+j)}(\lambda)
$$

for $m, j=1,2, \ldots$

\section{Numerical Example}

Based on the analysis above, we consider present the following example for computing the multivariate homogeneous matrix Padé approximants to the matrix function.

$$
F(x, y)=\left(\begin{array}{cc}
-(x y+1) e^{-x^{2}-y^{2}} & -\frac{3 y}{1+x^{2}+y^{2}} \\
\sqrt{4-x^{2}-y^{2}} & -x^{2}-y^{2}+\ln (1+x+y)
\end{array}\right)
$$

The exact value of $F$ at the point $\left(x_{0}, y_{0}\right)=\left(\frac{\sqrt{ }}{200}, \frac{1}{200}\right)$ is given by

$$
\begin{gathered}
F\left(x_{0}, y_{0}\right)=\left(\begin{array}{cc}
-\left(1+\frac{\sqrt{3}}{40000}\right) e^{10000} & -\frac{150}{10001} \\
\frac{\sqrt{ } \frac{}{39999}}{100} & -\frac{1}{10000}+\ln \left(\frac{201}{200}+\frac{\sqrt{3}}{200}\right)
\end{array}\right) \\
\approx\left(\begin{array}{cc}
-0.99994330194011203964 & -0.014998500149985001500 \\
1.9999749998437480468 & 0.013467793835957699252
\end{array}\right)
\end{gathered}
$$




\begin{tabular}{|c|c|c|}
\hline$m$ & {$[m-1 / m]_{F}\left(x_{0}, y_{0}\right)$} & $\left\|\left(F-[m-1 / m]_{F}\right)\left(x_{0}, y_{0}\right)\right\|_{2}$ \\
\hline 1 & $\left(\begin{array}{cc}-1 & 0 \\
2 & 0\end{array}\right)$ & 0.0201579 \\
\hline 2 & $\left(\begin{array}{cc}-0.999943304484753 & -0.014999149567271296 \\
1.999886608969506 & 0.013659479562703306\end{array}\right)$ & 0.000211085 \\
\hline 3 & $\left(\begin{array}{cc}-0.9999433069394953 & -0.014998500149985002 \\
1.99997500249975 & 0.013465606207034462\end{array}\right)$ & $2.18763 \times 10^{-6}$ \\
\hline 4 & $\left(\begin{array}{cc}-0.9999433019669471 & -0.01499850002173361 \\
1.9999749998580398 & 0.013467793874129514\end{array}\right)$ & $1.35594 \times 10^{-10}$ \\
\hline 5 & $\left(\begin{array}{cc}-0.9999433019401114 & -0.014998500147536242 \\
1.9999749998438265 & 0.013467793836360091\end{array}\right)$ & $2.48165 \times 10^{-12}$ \\
\hline 6 & $\left(\begin{array}{cc}-0.9999433019401119 & -0.014998500149985 \\
1.9999749998437488 & 0.013467793835961195\end{array}\right)$ & $3.6221 \times 10^{-15}$ \\
\hline 7 & $\left(\begin{array}{cc}-0.9999433019401117 & -0.014998500149985 \\
1.9999749998437482 & 0.01346779383595773\end{array}\right)$ & $3.33072 \times 10^{-16}$ \\
\hline 8 & $\left(\begin{array}{cc}-0.9999433019401123 & -0.014998500149985002 \\
1.999974999843748 & 0.013467793835957701\end{array}\right)$ & $3.17599 \times 10^{-17}$ \\
\hline
\end{tabular}

The exact value of $F$ at the point $\left(x_{0}, y_{0}\right)=\left(\frac{\overline{2}}{200}, \frac{\overline{2}}{200}\right)$ is given by

$$
\begin{gathered}
F\left(x_{0}, y_{0}\right)=\left(\begin{array}{cc}
-\frac{20001}{20000} e^{10000} & -\frac{150 \sqrt{2}}{10001} \\
\frac{\sqrt{39999}}{100} & -\frac{1}{10000}+\ln \left(1+\frac{1}{50 \overline{2}}\right)
\end{array}\right) \\
\approx\left(\begin{array}{cc}
-0.99995000000008332917 & -0.021211082327363689363 \\
1.9999749998437480468 & 0.013943068544592249131
\end{array}\right)
\end{gathered}
$$

\begin{tabular}{|c|c|c|}
\hline$m$ & {$[m-1 / m]_{F}\left(x_{0}, y_{0}\right)$} & $\left\|\left(F-[m-1 / m]_{F}\right)\left(x_{0}, y_{0}\right)\right\|_{2}$ \\
\hline 1 & $\left(\begin{array}{rr}-1 & 0 \\
2 & 0\end{array}\right)$ & 0.0253835 \\
\hline 2 & $\left(\begin{array}{cc}-0.999950002499875 & -0.021212142828455002 \\
1.99990000499975 & 0.014141428552303336\end{array}\right)$ & 0.000212066 \\
\hline 3 & $\left(\begin{array}{cc}-0.9999500049994999 & -0.021211082327363687 \\
1.9999750024997491 & 0.01394074154957599\end{array}\right)$ & $2.327 \times 10^{-6}$ \\
\hline 4 & $\left(\begin{array}{cc}-0.999950000022005 & -0.021211082118386446 \\
1.9999749998554406 & 0.013943068560980125\end{array}\right)$ & $2.10666 \times 10^{-10}$ \\
\hline 5 & $\left(\begin{array}{cc}-0.9999500000000815 & -0.02121108232250809 \\
1.9999749998438086 & 0.013943068544664601\end{array}\right)$ & $4.85614 \times 10^{-12}$ \\
\hline 6 & $\left(\begin{array}{cc}-0.9999500000000834 & -0.021211082327363698 \\
1.999974999843749 & 0.013943068544594393\end{array}\right)$ & $2.26543 \times 10^{-15}$ \\
\hline 7 & $\left(\begin{array}{cc}-0.9999500000000828 & -0.021211082327363687 \\
1.9999749998437477 & 0.01394306854459228\end{array}\right)$ & $7.11118 \times 10^{-16}$ \\
\hline 8 & $\left(\begin{array}{cc}-0.9999500000000833 & -0.021211082327363694 \\
1.9999749998437482 & 0.01394306854459225\end{array}\right)$ & $1.11328 \times 10^{-16}$ \\
\hline
\end{tabular}



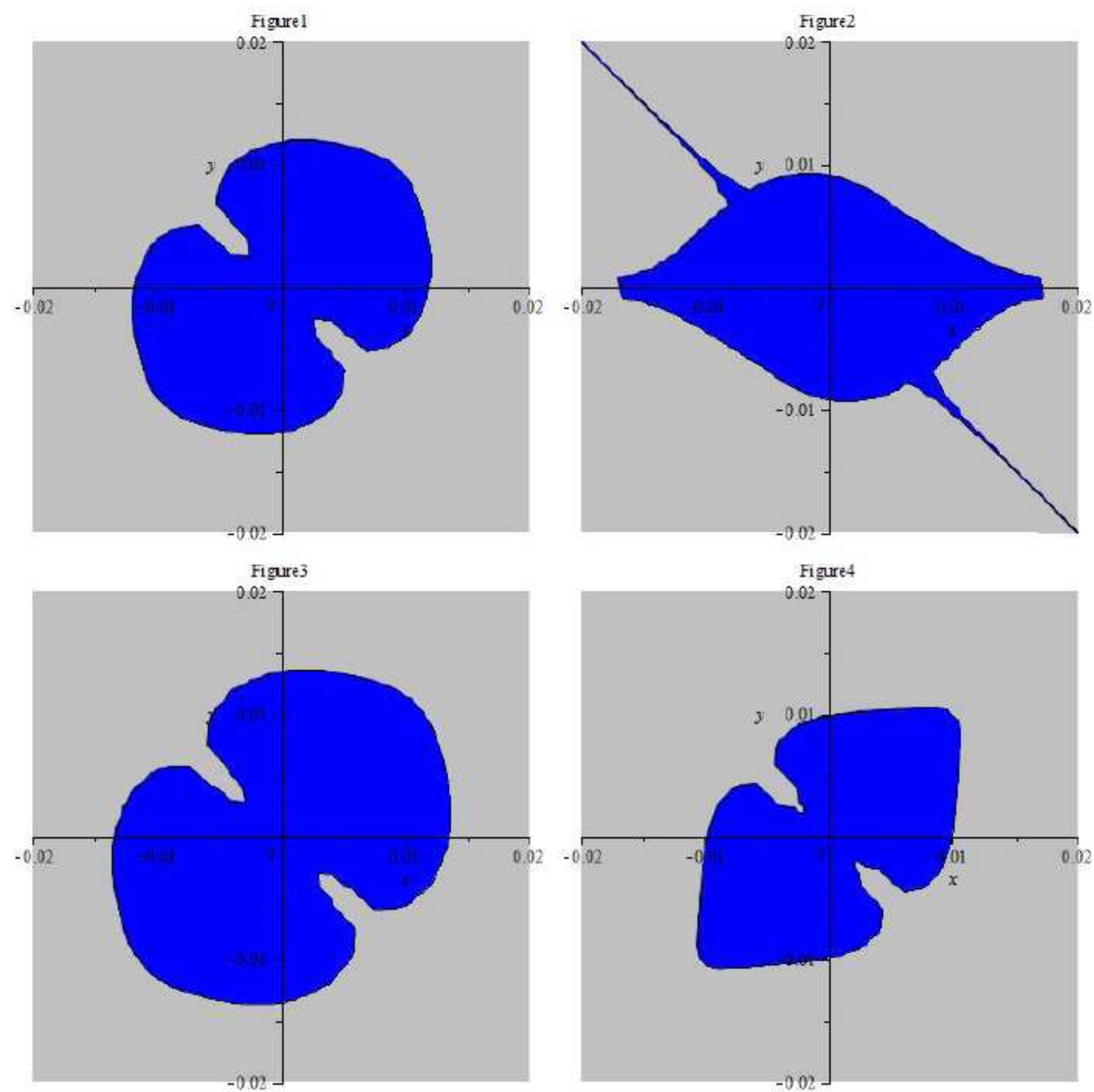

For $(x, y)$ varying over the domains represented by the figure $1-4$, the absolute values of all entries of the error matrix $\left(F-[3 / 4]_{F}^{H}\right)(x, y)$ are less than or equal to $10^{-10}$.

\section{References}

[1] J. Abouir, B. Benouahmane, A. Elbouchouki, A new family of multivariate 
matrix Padé approximants, Jean Jour. on Approximation, 6(2) (2014), $199-217$.

[2] S. Arioka, Padé-type approximants in multivariables, App. Numer. Math., 3(1987), 497-511.

[3] G. Baker, P.R. Graves-Morris, Padé Approximants, Encyclopedia of Mathematics and its Applications, Addison-Wesley Publishing Company, 13(1981), pp. 23-24.

[4] B. Benouahmane, A. Cuyt, Properties of multivariate homogeneous orthogonal polynomials, J. Approx. Theory, 113(2001), 1-20.

[5] C. Brezinski, Padé-type approximation and general orthogonal polynomials, Birkhauser Verlag, Basel, ISNM, 50(1980).

[6] N. K. Bose, S. Basu, Two-dimensional matrix Padé approximants: Existence, nonuniqueness and recursive computation, IEEE Trans. Autom. Control, AC-25, 3(1980), 509-514.

[7] A. Cuyt, Padé approximants for operators: theory and applications, SIAM J. Math. Anal., 14(1983), 195-202.

[8] A. Draux, Bibliographie-Index, Publication ANO 145. Lille, France, Univ. Lille, (1984).

[9] A. Draux, B. Moalla, Rectangular matrix Padé approximation and square matrix orthogonal polynomials, Numer. Algorithm, 14(1997), 321-341.

[10] C. Gu, Bivariate thiele-type matrix-valued rational interpolants, J. Comput. Appl. Math., 80(1997), 71-82.

[11] S. Kida, Padé-type and Padé approximants in several variables, Appl. Numer. Math., 6(1990), 371-391.

[12] C. Pestano-Gabino, C. Gongalez-Concepción, M. C. Farina-Gil, A type of matrix Padé approximant inspired by scalar component models, J. Comp. Appl. Math., 236(2012), 3360-3372.

[13] G. Xu, Existence and uniqueness of matrix Padé approximants, J. Comput. Math. 8(1990), 65-74.

[14] C. D. Zheng, R. H. Zhang, Generalized multivariate rectangular matrix Padé-type approximants, IEEE Trans. Cir. and Sys., 54 (9) (Sep. 2007), 2099-2105. 
[15] C. D. Zheng, H. Zhang, H. Tian, Generalized Homogeneous multivariate matrix Padé-type approximants and Padé approximants, IEEE Trans. Autom. Cont., 52(11) (Nov. 2007), 2160-2165. 